\title{
MULTI-TILING AND EQUIDECOMPOSABILITY OF POLYTOPES BY LATTICE TRANSLATES
}

\author{
NIR LEV AND BOCHEN LIU
}

\begin{abstract}
We characterize the polytopes in $\mathbb{R}^{d}$ (not necessarily convex or connected ones) which multi-tile the space by translations along a given lattice. We also give a necessary and sufficient condition for two polytopes in $\mathbb{R}^{d}$ to be equidecomposable by lattice translations.
\end{abstract}

\section{IntRoduction}

A simplex in $\mathbb{R}^{d}$ is the convex hull of $d+1$ points which do not all lie in some hyperplane. By a polytope in $\mathbb{R}^{d}$ we mean a set which can be represented as the union of a finite number of simplices with disjoint interiors. Remark that a polytope is not necessarily a convex, nor even a connected, set.

By a lattice in $\mathbb{R}^{d}$ we refer to a discrete subgroup generated by $d$ linearly independent vectors.

1.1. Let $A$ be a polytope in $\mathbb{R}^{d}$, and $L$ be a lattice in $\mathbb{R}^{d}$. The polytope $A$ is said to $k$-tile by translations with respect to $L$ (where $k$ is a positive integer) if almost every point of the space $\mathbb{R}^{d}$ lies in exactly $k$ among the sets $A+\lambda(\lambda \in L)$, that is,

$$
\sum_{\lambda \in L} \chi_{A}(x-\lambda)=k \quad \text { a.e. }
$$

where $\chi_{A}$ is the indicator function of $A$. In this case, the number $k$ is called the level, or the multiplicity, of the tiling.

It is well-known that the condition $\operatorname{vol}(A)=k \operatorname{det}(L)$ is necessary for $k$-tiling by translations of $A$ along $L$, where $\operatorname{vol}(A)$ denotes the volume of $A$, and $\operatorname{det}(L)$ is the volume of some (or equivalently, of any) fundamental parallelepiped of the lattice $L$.

We are concerned with the following problem:

Given a polytope $A$ and a lattice $L$, formulate in effective terms a condition which is necessary and sufficient for the translates of $A$ along $L$ to be a $k$-tiling.

This problem was studied for some special classes of polytopes, by several authors. For example, if $A$ is a convex polygon in two dimensions, then a necessary and sufficient condition for $k$-tiling was given by Bolle Bol94. Kolountzakis Kol00] extended this result for a wider class of planar polygons, which includes also non-convex ones. Gravin, Robins and Shiryaev proved in GRS12 that for a polytope $A \subset \mathbb{R}^{d}$ to $k$-tile by translations along a lattice $L$, it is sufficient that $A$ be centrally symmetric, have centrally symmetric facets, and that all the vertices of $A$ lie in $L$. It was also proved in

Date: July 29, 2019.

2010 Mathematics Subject Classification. 52B11, 52B45, 52C22.

Key words and phrases. Polytopes, multi-tiling, equidecomposability, Hilbert's third problem.

Research supported by ISF grant No. 227/17 and ERC Starting Grant No. 713927. 
GRS12 that if $A$ is convex, then the first two conditions, namely that $A$ be centrally symmetric and have centrally symmetric facets, are also necessary for $k$-tiling.

In this paper, we give a complete characterization of the polytopes $A \subset \mathbb{R}^{d}$ (not necessarily convex or connected ones) which tile at some level $k$ by translations along a lattice $L$. We formulate this characterization in terms of Hadwiger functionals, whose definition will now be given.

1.2. Let $r$ be an integer, $0 \leqslant r \leqslant d-1$, and suppose that

$$
V_{r} \subset V_{r+1} \subset \cdots \subset V_{d-1} \subset V_{d}=\mathbb{R}^{d}
$$

is a sequence of affine subspaces such that $V_{j}$ has dimension $j$ (where an affine subspace means a translated linear subspace). Each subspace $V_{j}(r \leqslant j \leqslant d-1)$ in the sequence divides the next one $V_{j+1}$ into two half-spaces; we call one of them the positive halfspace, and the other the negative half-space. Such a sequence of nested affine subspaces, endowed with a choice of positive and negative half-spaces, will be called an $r$-flag, and will be denoted by $\Phi$.

Let $A$ be a polytope in $\mathbb{R}^{d}$, and suppose that $A$ has a sequence of faces

$$
F_{r} \subset F_{r+1} \subset \cdots \subset F_{d-1} \subset F_{d}=A,
$$

where $F_{j}$ is a $j$-dimensional face of $A$ which is contained in $V_{j}(r \leqslant j \leqslant d-1)$. To each face $F_{j}$ we assign a coefficient $\varepsilon_{j}$, given by $\varepsilon_{j}=+1$ if $F_{j+1}$ adjoins $V_{j}$ from the positive side, and $\varepsilon_{j}=-1$ if $F_{j+1}$ adjoins $V_{j}$ from the negative side. We then define

$$
\omega_{\Phi}(A)=\sum \varepsilon_{r} \varepsilon_{r+1} \cdots \varepsilon_{d-1} \operatorname{Vol}_{r}\left(F_{r}\right),
$$

where the sum goes through all sequences of faces of $A$ with the property above, and where $\operatorname{Vol}_{r}\left(F_{r}\right)$ denotes the $r$-dimensional volume of $F_{r}$. If no such a sequence of faces of $A$ exists, then we let $\omega_{\Phi}(A)=0$. In the case when $r=0$, by a 0 -dimensional face we mean a vertex of $A$, and its 0 -dimensional volume is defined to be 1 .

Finally, let $L$ be a lattice in $\mathbb{R}^{d}$. For each $r$-flag $\Phi(0 \leqslant r \leqslant d-1)$ we define

$$
H_{\Phi}(A, L)=\sum_{\Psi} \omega_{\Psi}(A)
$$

where $\Psi$ runs through all $r$-flags for which there exists $\lambda \in L$ such that $\Psi$ can be obtained from $\Phi$ by translating all the affine subspaces in (1.2), as well as the positive and negative half-spaces, by the vector $\lambda$. (Remark that each $r$-flag $\Psi$ in the sum is taken into account only once, even if there is more than a single vector $\lambda \in L$ which carries $\Phi$ onto $\Psi$.) Notice that there can be only finitely many nonzero terms in the sum (1.5), hence this sum has a well-defined value. We will call $H_{\Phi}$ the Hadwiger functional associated to the $r$-flag $\Phi$.

We can now state our result that characterizes the polytopes $A$ whose translates along a lattice $L$ form a $k$-tiling:

Theorem 1.1. Let $A$ be a polytope in $\mathbb{R}^{d}$, and $L$ be a lattice in $\mathbb{R}^{d}$. Then $A$ tiles at some level $k$ by translations with respect to $L$, if and only if

$$
H_{\Phi}(A, L)=0
$$

for every $r$-flag $\Phi(0 \leqslant r \leqslant d-1)$. 
Notice that in order to check the condition in this result, it is actually enough to verify (1.6) for finitely many flags $\Phi 1$ Hence, only a finite number of verifications is in fact needed. Moreover, the value of each $H_{\Phi}(A, L)$ depends on $A$ and $L$ in an elementary way. So the condition in Theorem 1.1 can be effectively checked in concrete situations.

In Section 2, we will illustrate the use of Theorem 1.1 by analyzing some examples. In particular, the above mentioned results from [Bol94], [Kol00] and [GRS12] will be recovered based on Theorem 1.1.

1.3. The $k$-tiling problem is closely related to another subject in discrete geometry, namely, the theory of equidecomposability of polytopes by rigid motions. This is a classical subject, which goes back to Hilbert's third problem (one of the famous 23 problems posed by Hilbert at the International Congress of Mathematicians in 1900).

If $A$ and $B$ are two polytopes in $\mathbb{R}^{d}$, then they are said to be equidecomposable (or dissection equivalent, or scissors congruent) if the polytope $A$ can be partitioned, up to measure zero, into a finite number of smaller polytopes which can be rearranged using rigid motions to form, again up to measure zero, a partition of the polytope $B$.

It is obvious that if two polytopes $A$ and $B$ are equidecomposable, then they must have the same volume. Hilbert's third problem was concerned with the converse assertion: are any two polytopes $A$ and $B$ of the same volume equidecomposable? It has been known earlier that in two dimensions, any two polygons of equal area are equidecomposable, but in the same year 1900 it was shown by Dehn that in three dimensions such a result is no longer true (see the book [Bol78] for a comprehensive exposition).

The problem of equidecomposability of polytopes has also been studied when extra restrictions are imposed on the way in which the pieces of the partition are allowed to be rearranged. If $G$ is a group of rigid motions of $\mathbb{R}^{d}$, then two polytopes $A$ and $B$ are said to be $G$-equidecomposable if $A$ can be partitioned into a finite number of polytopes which can be rearranged, using only motions from $G$, to form a partition of $B$ (where as before, the pieces of each partition may overlap but only up to measure zero).

In this paper, we consider the following question:

Given two polytopes $A, B$ and a lattice $L$, under what condition (that can be checked effectively) are $A$ and $B$ equidecomposable using only translations by vectors from $L$ ?

Our next result characterizes precisely when such an equidecomposition exists. To state the result, we again use the Hadwiger functionals $H_{\Phi}$ described above.

Theorem 1.2. Let $A$ and $B$ be two polytopes in $\mathbb{R}^{d}$, and let $L$ be a lattice in $\mathbb{R}^{d}$. Then $A$ and $B$ are equidecomposable with respect to translations by vectors from $L$, if and only if $A$ and $B$ have the same volume and

$$
H_{\Phi}(A, L)=H_{\Phi}(B, L)
$$

for every $r$-flag $\Phi(0 \leqslant r \leqslant d-1)$.

As was the case with Theorem 1.1, also the condition in this result is elementary enough so that it can be effectively checked in concrete situations.

\footnotetext{
${ }^{1}$ Indeed, it is enough to verify (1.6) only for flags $\Phi$ that can be obtained from some sequence of faces as in (1.3), by taking each subspace $V_{j}$ to be the affine hull of the face $F_{j}(r \leqslant j \leqslant d-1)$. So the number of verifications needed is bounded by the number of such sequences of faces.
} 
1.4. In order to understand the role played by the Hadwiger functionals in the theory of equidecomposability of polytopes, it is necessary to be acquainted with an important notion in this theory, namely, the notion of additive invariants.

Let $G$ be a group of rigid motions of $\mathbb{R}^{d}$. A function $\varphi$, defined on the set of all polytopes in $\mathbb{R}^{d}$, is said to be an additive $G$-invariant if (i) it is additive, namely, if $A$ and $B$ are two polytopes with disjoint interiors then $\varphi(A \cup B)=\varphi(A)+\varphi(B)$; and (ii) it is invariant under motions from the group $G$, that is, $\varphi(A)=\varphi(g(A))$ whenever $A$ is a polytope and $g \in G$.

It is obvious that for two polytopes $A$ and $B$ to be $G$-equidecomposable, a necessary condition is that $\varphi(A)=\varphi(B)$ for any additive $G$-invariant $\varphi$. A general problem is to construct a "complete system" of additive $G$-invariants, that is, invariants which together provide a condition which is both necessary and sufficient for two polytopes of the same volume to be $G$-equidecomposable.

In his solution to Hilbert's third problem, Dehn constructed an additive invariant with respect to the group of all rigid motions of $\mathbb{R}^{3}$, which allowed him to show that a regular tetrahedron and a cube of the same volume are not equidecomposable [Deh01]. Dehn invariants for polytopes in $\mathbb{R}^{d}$ have also been studied [Had54], and shown to form a complete system in dimensions $d=3,4$ [Syd65, Jes72]. It remains an open problem as to whether these invariants are complete also in dimensions $d \geqslant 5$.

Additive invariants with respect to the group of translations of $\mathbb{R}^{d}$ were introduced by Hadwiger [Had52, Had57]. It was proved that these invariants form a complete system, so that together they provide a necessary and sufficient condition for two polytopes of the same volume to be equidecomposable by translations. This was proved by Hadwiger and Glur in dimension two [HG51, by Hadwiger in dimension three [Had68, and by Jessen and Thorup [JT78], and independently Sah [Sah79], in every dimension.

The question which concerns us in this paper, involves equidecomposability with respect to the group of translations by vectors belonging to a lattice $L$. How to construct additive invariants with respect to this group of motions? We can use the fact (observed by Hadwiger) that if $\Phi$ is an $r$-flag $(0 \leqslant r \leqslant d-1)$, then the function $\omega_{\Phi}$ defined by (1.4) is an additive function on the set of all polytopes in $\mathbb{R}^{d}$ (see e.g. Sah79, Section 2.6]). Hence the Hadwiger functional $H_{\Phi}(\cdot, L)$ defined by (1.5) is easily seen to be an additive invariant with respect to translations by vectors from $L$.

In fact, this construction is valid for any subgroup $L$ of $\mathbb{R}^{d}$ (that is, not only for a lattice). In particular, if $L=\mathbb{R}^{d}$, then the obtained invariants are the classical ones introduced by Hadwiger. In the case when $L$ is a proper subgroup of $\mathbb{R}^{d}$, the invariants $H_{\Phi}(\cdot, L)$ were first considered in [GL15, Section 5].

This clarifies why the "only if" part of Theorem 1.2 is true. Indeed, for two polytopes $A$ and $B$ to be equidecomposable using translations by vectors from $L$, it is necessary that $\varphi(A)=\varphi(B)$ for any additive function $\varphi$ which is invariant with respect to translations by vectors from $L$ (and in particular, $A$ and $B$ must have the same volume). In fact, the same is true for any subgroup $L$ of $\mathbb{R}^{d}$, not necessarily a lattice.

It is therefore the "if" part of Theorem 1.2 which constitutes the main result. The result can be equivalently stated by saying that if $L$ is a lattice, then the Hadwiger functionals $H_{\Phi}(\cdot, L)$ form a complete system of invariants with respect to the group of translations by vectors from $L$. Our proof of this result is based on completely different considerations than the proofs in [HG51, Had68, JT78, Sah79], where it is shown that 
the classical Hadwiger invariants $H_{\Phi}\left(\cdot, \mathbb{R}^{d}\right)$ are complete with respect to the group of all the translations of $\mathbb{R}^{d}$.

The question as to whether the Hadwiger invariants $H_{\Phi}(\cdot, L)$ are complete when $L$ is a proper subgroup of $\mathbb{R}^{d}$, was raised in [GL15, Section 7.1]. Theorem 1.2 provides an affirmative answer to this question in the case when $L$ is a lattice. For a general subgroup $L$, the problem remains open.

1.5. In all that has been said so far, we have still not explained what is the relation between the two problems that have been discussed, that is, the $k$-tiling problem and the equidecomposability problem, both considered with respect to lattice translates.

The following elementary fact demonstrates the relation between these two problems:

Proposition 1.3. Let $A$ and $B$ be two polytopes in $\mathbb{R}^{d}$, and let $L$ be a lattice in $\mathbb{R}^{d}$. Assume that $B$ tiles at some level $k$ by translations along $L$. Then the translates of $A$ along $L$ is also a $k$-tiling, if and only if $A$ and $B$ are equidecomposable with respect to translations by vectors from $L$.

An interesting case to which this proposition applies is when $B$ is the union of $k$ disjoint fundamental parallelepipeds of the lattice $L$. It is obvious that such a polytope $B$ tiles at level $k$ by translations along $L$. It is also easy to check that $H_{\Phi}(B, L)=0$ for every $\Phi$. Hence, one can see that Theorem 1.2 and Proposition 1.3 imply the "only if" part of Theorem 1.1, namely, for a polytope $A$ to $k$-tile by translations along a lattice $L$, it is necessary that $H_{\Phi}(A, L)=0$ for every $\Phi$.

On the other hand, the "if" part of Theorem 1.1 does not follow from Theorem 1.2 and Proposition 1.3 in a similar way. The missing ingredient which is needed for this purpose, is a result which states that the vanishing of all the Hadwiger functionals $H_{\Phi}(A, L)$ implies that the volume of $A$ must be an integral multiple of $\operatorname{det}(L)$.

In order to prove Theorems 1.1 and 1.2, it is natural to realize the set of all polytopes as a subset of a certain group, called the polytope group in $\mathbb{R}^{d}$. We will prove a theorem, which is in fact the main result of this paper (Theorem 4.1), that characterizes the elements of the polytope group in $\mathbb{R}^{d}$ whose translates along a lattice $L$ form a $k$-tiling. Theorems 1.1 and 1.2 will be obtained as consequences of this result.

\section{EXAMPLES}

In this section we consider some specific classes of polytopes, and obtain conditions for the polytopes in these classes to $k$-tile by lattice translates. The examples include finite unions of intervals in dimension one, convex polytopes in dimensions two and three, and some other classes.

In analyzing these examples, we will illustrate how to use the characterization given in Theorem 1.1 of the polytopes which $k$-tile by lattice translates. In particular we will recover the results from [Bol94], Kol00] and GRS12] mentioned above.

Similar examples were analyzed in [GL15, Section 5], where the Hadwiger functionals were studied not with respect to lattice translations, but with respect to translations along a countable, dense subgroup of $\mathbb{R}^{d}$ generated by $d+1$ vectors. Some of the results of this section are analogous to those discussed in [GL15. 
2.1. The simplest situation to which Theorem 1.1 applies, takes place in dimension one, where a "polytope" $A$ is a finite union of disjoint closed intervals $\left[a_{j}, b_{j}\right]$, and a "lattice" $L$ is an arithmetic progression passing through the origin.

In dimension one, a 0 -flag $\Phi$ is determined by a single point $x$, that divides $\mathbb{R}$ into a positive and a negative half-line. The value of the Hadwiger functional $H_{\Phi}(A, L)$ is then equal to the difference between the number of left endpoints $a_{j}$, and the number of right endpoints $b_{j}$, which belong to the set $L+x$. Hence, it follows from Theorem 1.1 that for $A$ to tile at some level $k$ by translations with respect to $L$, it is necessary and sufficient that any translate of $L$ contain the same number of left and right endpoints.

This condition can be equivalently stated as follows:

Corollary 2.1. Let $A \subset \mathbb{R}$ be the union of $n$ disjoint intervals $\left[a_{j}, b_{j}\right], 1 \leqslant j \leqslant n$, and let $L$ be a lattice in $\mathbb{R}$. Then $A$ tiles at some level $k$ by translations along $L$, if and only if there exists a permutation $\sigma$ of $\{1,2, \ldots, n\}$ such that $b_{\sigma(j)}-a_{j} \in L$ for every $j$.

2.2. In our next example, we consider the case when $A$ is a two-dimensional convex polygon. In this case, we will see that Theorem 1.1 recovers the main result from [Bol94]. The result can be stated the following way:

Corollary 2.2 (Bolle [Bol94]). Let $A$ be a convex polygon in $\mathbb{R}^{2}$, and $L$ be a lattice in $\mathbb{R}^{2}$. Then $A$ tiles at some level $k$ by translations with respect to $L$, if and only if $A$ is centrally symmetric, and for each pair of parallel edges $e$ and $e^{\prime}$ of $A$ the following two conditions are satisfied:

(i) for some $\lambda \in L$, both $e+\lambda$ and $e^{\prime}$ lie on the same line;

(ii) if the vector $\vec{e}$ does not belongs to $L$, then the translation vector which carries $e$ onto $e^{\prime}$, is in $L$.

Proof. By Theorem 1.1 it will be enough to show that the condition that $A$ is centrally symmetric and satisfies (i) and (ii) for any pair of parallel edges $e$ and $e^{\prime}$, is necessary and sufficient for the vanishing of all the Hadwiger functionals $H_{\Phi}(A, L)$. Below we will prove the necessity of the condition. The sufficiency can be proved in a similar way.

We therefore assume that $H_{\Phi}(A, L)=0$ for all 0 - and 1-flags $\Phi$. Let $e$ be any edge of $A$, and let $\Phi$ be a 1 -flag determined by the line $\ell$ containing $e$. Since $A$ is convex, there can be at most one other edge $e^{\prime}$ which is parallel to $e$. The condition $H_{\Phi}(A, L)=0$ guarantees that such an edge $e^{\prime}$ indeed exists, and that $e^{\prime}$ is of the same length as $e$. Since this is true for every edge $e$ of $A$, we conclude that $A$ is centrally symmetric. Moreover, for any edge $e$, the line $\ell^{\prime}$ containing the parallel edge $e^{\prime}$ must be of the form $\ell^{\prime}=\ell+\lambda$ for some $\lambda \in L$, which implies condition (i).

To obtain condition (ii) as well, we again let $e$ be one of the edges of $A$. Let $p$ be one of the endpoints of $e$, and $p^{\prime}$ be the endpoint of the parallel edge $e^{\prime}$ that is given by $p^{\prime}=p+\tau$, where $\tau$ is the translation vector which carries $e$ onto $e^{\prime}$ (see Figure 2.1). Let $\Phi$ be a 0 -flag determined by the point $p$ and the line $\ell$ which contains the edge $e$. Then for the condition $H_{\Phi}(A, L)=0$ to hold, the contribution of the point $p$ to the sum (1.5) must be cancelled by another term in the sum, which necessarily corresponds to a vertex of $A$ which lies in the set $L+p$, and moreover this vertex can be either the other endpoint of $e$ or the vertex $p^{\prime}$. This shows that if the vector $\vec{e}$ is not in $L$, then $\tau$ must be in $L$, which establishes condition (ii) and thus concludes the proof. 


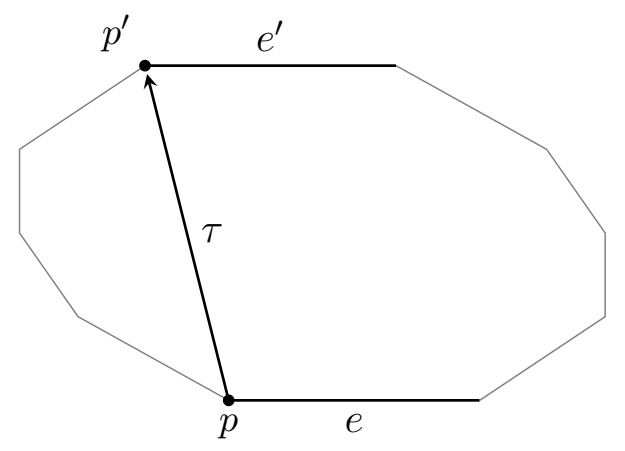

Figure 2.1. The convex polygon $A$ in the proof of Corollary 2.2 .

2.3. Actually, the convexity assumption was not essential in the proof of Corollary 2.2. The main role of this assumption was to ensure that for each edge $e$ of $A$ there can be at most one other edge $e^{\prime}$ which is parallel to $e$. Hence, basically the same proof is valid in this more general situation. In this case, Theorem 1.1 recovers the following generalization of Corollary 2.2, which is due to Kolountzakis [Kol00, Theorem 2]:

Corollary 2.3 (Kolountzakis [Kol00]). Let $A$ be a polygon in $\mathbb{R}^{2}$ with the property that for each edge $e$ of $A$, there is at most one other edge $e^{\prime}$ which is parallel to $e$. Then $A$ tiles at some level $k$ by translations with respect to a lattice $L$ in $\mathbb{R}^{2}$, if and only if for each edge $e$ of $A$ the following three conditions are satisfied: $(i)$ there exists an edge $e^{\prime}$ which is parallel to $e$, and $e^{\prime}$ has the same length as $e ;($ ii) for some $\lambda \in L$, both $e+\lambda$ and $e^{\prime}$ lie on the same line; and (iii) if the vector $\vec{e}$ does not belongs to $L$, then the translation vector which carries e onto $e^{\prime}$, is in $L$.

2.4. For $k$-tiling by translates of a convex polytope in higher dimensions, the following result was obtained in [GRS12, Theorem 1]: If $A \subset \mathbb{R}^{d}$ is a convex polytope which $k$-tiles by translations, then $A$ is centrally symmetric and has centrally symmetric facets.

(Recall that a facet of a polytope $A \subset \mathbb{R}^{d}$ is a $(d-1)$-dimensional face of $A$.)

Notice that this result is not specific for $k$-tilings by lattice translates; the conclusion is true regardless of whether the translation set is a lattice or not.

The proof of the result consists of two parts. In the first part, it is shown that the $k$-tiling assumption implies that the (classical) Hadwiger functionals $H_{\Phi}\left(A, \mathbb{R}^{d}\right)$ (that are invariant with respect to all translations, not only lattice translations) vanish for all $r$-flags $\Phi(0 \leqslant r \leqslant d-1)$. In the special case of $k$-tiling with respect to a lattice $L$, this can be deduced from Theorem [1.1, since the condition that $H_{\Phi}(A, L)=0$ for every $\Phi$, implies in particular that also $H_{\Phi}\left(A, \mathbb{R}^{d}\right)=0$ for every $\Phi$. In the general case (that is, for $k$-tiling with respect to an arbitrary translation set), the vanishing of the Hadwiger functionals is proved using a volume growth argument, see [Mür75], [LM95a, Theorem 3], GRS12, Lemma 3.3]. In fact, this part of the proof does not require the polytope $A$ to be convex; the same argument is valid for an arbitrary polytope $A \subset \mathbb{R}^{d}$.

In the second part of the proof, the vanishing of the Hadwiger functionals $H_{\Phi}\left(A, \mathbb{R}^{d}\right)$ is shown to imply that the convex polytope $A$ must be centrally symmetric and have centrally symmetric facets, see [Mür77, Section 3.3], GRS12, pp. 640-641]. This is proved based on a classical theorem due to Minkowski, which states that a convex 
polytope $A \subset \mathbb{R}^{d}$ is centrally symmetric if and only if every facet of $A$ has a parallel facet of the same $(d-1)$-dimensional volume.

2.5. Next, we consider convex polytopes in three-dimensional space. In this case, we will use Theorem 1.1 to obtain a condition which is necessary and sufficient for $k$-tiling by lattice translates, similar in spirit to Bolle's criterion in two dimensions.

To state the result, we will use the terminology from [GKRS13] where the notion of a four-legged-frame was defined. The definition of a four-legged-frame will now be given.

Assume that $A \subset \mathbb{R}^{3}$ is a convex polytope, which is centrally symmetric and has centrally symmetric facets (as we have seen above, this condition is necessary for $A$ to $k$-tile by translations). Let $F$ be one of the facets of $A$. Then, due to the central symmetry assumptions, $F$ has a parallel facet $F^{\prime}$ which is a translate of $F$. Let $\tau^{\prime}$ denote the translation vector which carries $F$ onto $F^{\prime}$. Further, let $e$ be an edge of $A$ which is contained in $F$. Then the central symmetry of $F$ implies that there is another edge $e^{\prime \prime}$ of $F$, which is parallel to $e$ and has the same length. Let $\tau^{\prime \prime}$ be the translation vector which carries $e$ onto $e^{\prime \prime}$. Finally, consider the four parallel edges of $A$ given by

$$
e, \quad e^{\prime}=e+\tau^{\prime}, \quad e^{\prime \prime}=e+\tau^{\prime \prime}, \quad e^{\prime \prime \prime}=e+\tau^{\prime}+\tau^{\prime \prime},
$$

see the illustration in Figure 2.2. Any system $\left(e, e^{\prime}, e^{\prime \prime}, e^{\prime \prime \prime}\right)$ of four edges of $A$ which can be obtained in this way, will be called (following [GKRS13]) a four-legged-frame of $A$.

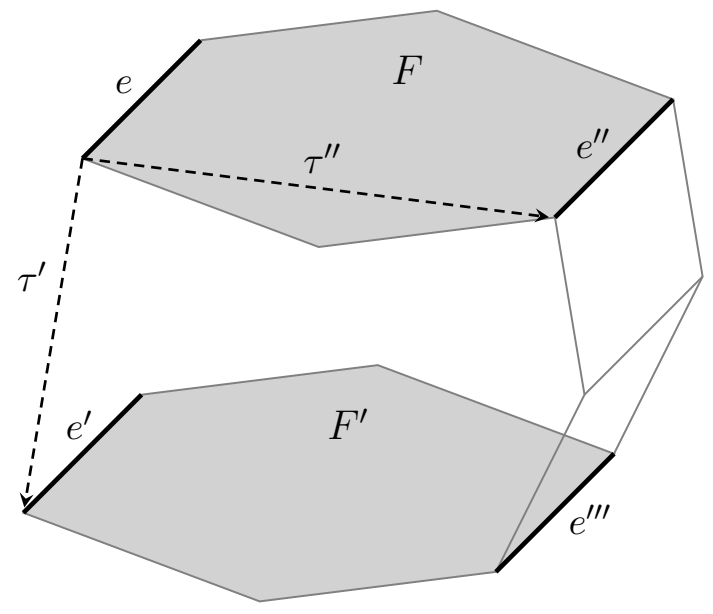

Figure 2.2. A four-legged-frame $\left(e, e^{\prime}, e^{\prime \prime}, e^{\prime \prime \prime}\right)$ of a convex polytope in $\mathbb{R}^{3}$ that is centrally symmetric and has centrally symmetric facets.

We can now state our characterization of the three-dimensional convex polytopes which $k$-tile by translations along a lattice:

Theorem 2.4. Let $A$ be a convex polytope in $\mathbb{R}^{3}$, and $L$ be a lattice in $\mathbb{R}^{3}$. Then $A$ tiles at some level $k$ by translations with respect to $L$, if and only if $A$ is centrally symmetric, $A$ has centrally symmetric facets, and for each four-legged-frame $\left(e, e^{\prime}, e^{\prime \prime}, e^{\prime \prime \prime}\right)$ of $A$ the following three conditions are satisfied:

(i) for some vector $\lambda^{\prime} \in L$, both $F+\lambda^{\prime}$ and $F^{\prime}$ lie on the same plane;

(ii) there is $\lambda \in L$ such that $e+\lambda$ lies on the same line with either $e^{\prime}$ or $e^{\prime \prime}$; 
(iii) if no one of the three vectors $\vec{e}, \tau^{\prime}, \tau^{\prime \prime}$ belongs to $L$, then all the four vectors of the form $\vec{e} \pm \tau^{\prime} \pm \tau^{\prime \prime}$ (corresponding to all possible choices of signs) are in L.

The facets $F, F^{\prime}$ and the vectors $\tau^{\prime}, \tau^{\prime \prime}$ which appear in conditions (i) and (iii) correspond to the four-legged-frame $\left(e, e^{\prime}, e^{\prime \prime}, e^{\prime \prime \prime}\right)$ in the way described above.

Proof. By Theorem 1.1 it will be enough to show that the above conditions are necessary and sufficient for the vanishing of all the Hadwiger functionals $H_{\Phi}(A, L)$. Below we will prove the necessity of these conditions. The sufficiency can be proved in a similar way.

We therefore assume that $H_{\Phi}(A, L)=0$ for all 0-, 1- and 2-flags $\Phi$. Then we know that $A$ must be centrally symmetric and have centrally symmetric facets (see Section 2.4). Let $\left(e, e^{\prime}, e^{\prime \prime}, e^{\prime \prime \prime}\right)$ be a four-legged-frame of $A$, and let the facets $F, F^{\prime}$ and the vectors $\tau^{\prime}, \tau^{\prime \prime}$ correspond to $\left(e, e^{\prime}, e^{\prime \prime}, e^{\prime \prime \prime}\right)$ as described above.

Let $\Phi$ be a 2-flag determined by the plane $P$ that contains the facet $F$. From the condition $H_{\Phi}(A, L)=0$ we infer that the parallel plane $P^{\prime}$ which contains the facet $F^{\prime}$ must be of the form $P^{\prime}=P+\lambda^{\prime}$ for some $\lambda^{\prime} \in L$, which yields (i),

Next, we let $\Phi$ be a 1 -flag determined by the line $\ell$ that contains the edge $e$, and by the plane $P$ containing the facet $F$. For the condition $H_{\Phi}(A, L)=0$ to hold, the contribution of the edge $e$ to the sum (1.5) must be cancelled by another term in the sum, which necessarily corresponds to another edge of $A$ that lies in some line of the form $\ell+\lambda, \lambda \in L$, and this edge can be either $e^{\prime}$ or $e^{\prime \prime}$. Thus we obtain (ii),

Finally, let $p$ and $q$ denote the two endpoints of the edge $e$. We divide the eight endpoints of the four edges $e, e^{\prime}, e^{\prime \prime}, e^{\prime \prime \prime}$ into two subsets with four elements each,

$$
p, \quad q+\tau^{\prime}, \quad q+\tau^{\prime \prime}, \quad p+\tau^{\prime}+\tau^{\prime \prime},
$$

and

$$
q, \quad p+\tau^{\prime}, \quad p+\tau^{\prime \prime}, \quad q+\tau^{\prime}+\tau^{\prime \prime}
$$

Let $\Phi$ be a 0 -flag that is determined by some point $x \in \mathbb{R}^{3}$, by a line $\ell$ through $x$ that is parallel to $e$, and by a plane $P$ containing $\ell$ which is parallel to $F$. Then the value of $H_{\Phi}(A, L)$ is equal to the difference between the number of vertices from the subset (2.1) that belong to $L+x$, and the number of vertices from subset (2.2) that are in $L+x$. One can verify that for the condition $H_{\Phi}(A, L)=0$ to hold regardless of the choice of the point $x$, it is necessary and sufficient that at least one of the three vectors $\vec{e}, \tau^{\prime}, \tau^{\prime \prime}$ belong to $L$, or that all the four vectors of the form $\vec{e} \pm \tau^{\prime} \pm \tau^{\prime \prime}$ be in $L$. This implies (iii), and concludes the proof.

2.6. As our last example, we will use Theorem 1.1 to recover [GRS12, Theorem 2], which gives a useful sufficient condition for a polytope $A \subset \mathbb{R}^{d}$ to $k$-tile by lattice translates. Remark that $A$ is not assumed to be convex, or connected, in this result.

Corollary 2.5 (Gravin, Robins, Shiryaev [GRS12]). Let $A$ be a polytope in $\mathbb{R}^{d}$, and L be a lattice in $\mathbb{R}^{d}$. Suppose that $A$ is centrally symmetric, $A$ has centrally symmetric facets, and that all the vertices of $A$ lie in $L$. Then the translates of $A$ with respect to $L$ form a $k$-tiling for some $k$.

Proof. Let $t$ denote the center of symmetry of $A$, that is, $t$ is the unique point such that $A=-A+2 t$. If $F$ is one of the facets of $A$, then by reflecting $F$ through the point $t$ we obtain another facet $F^{\prime}:=-F+2 t$ of $A$. Let $t^{\prime}$ denote the center of symmetry of the facet $F$, so that we have $F=-F+2 t^{\prime}$. It follows that $F^{\prime}=F+2\left(t-t^{\prime}\right)$. Since all 
the vertices of $A$ lie in $L$, the translation vector $\lambda:=2\left(t-t^{\prime}\right)$ which carries $F$ onto $F^{\prime}$, must belong to $L$. Moreover, as $A$ and $F$ cannot have the same center of symmetry, we have $t \neq t^{\prime}$, and therefore $\lambda \neq 0$. Hence $F$ and $F^{\prime}$ are two distinct facets of $A$.

We thus conclude that the collection of all the facets of $A$ can be partitioned into pairs of parallel facets, such that if two facets $F$ and $F^{\prime}$ constitute one of these pairs then $F^{\prime}$ can be obtained from $F$ by reflection through the center of symmetry of $A$, and in addition there is a nonzero vector $\lambda \in L$ such that $F^{\prime}=F+\lambda$. We will show that this implies that $H_{\Phi}(A, L)=0$ for every $r$-flag $\Phi(0 \leqslant r \leqslant d-1)$. Due to Theorem 1.1, this suffices in order to establish that $A$ tiles at some level $k$ by translations along $L$.

Let $0 \leqslant r \leqslant d-1$, and let

$$
F_{r} \subset F_{r+1} \subset \cdots \subset F_{d-1} \subset F_{d}=A
$$

be a sequence of faces of $A$, such that $F_{j}$ has dimension $j(r \leqslant j \leqslant d-1)$. The facet $F_{d-1}$ from this sequence belongs to one of the pairs of facets described above, so it is in the same pair with another facet $F_{d-1}^{\prime}$. Let $\lambda \in L$ be the nonzero translation vector such that $F_{d-1}^{\prime}=F_{d-1}+\lambda$. Then we can define another sequence of faces

$$
F_{r}^{\prime} \subset F_{r+1}^{\prime} \subset \cdots \subset F_{d-1}^{\prime} \subset F_{d}^{\prime}=A
$$

given by $F_{j}^{\prime}:=F_{j}+\lambda(r \leqslant j \leqslant d-1)$.

Suppose that $\Psi$ is an $r$-flag determined by a sequence

$$
V_{r} \subset V_{r+1} \subset \cdots \subset V_{d-1} \subset V_{d}=\mathbb{R}^{d},
$$

such that $V_{j}$ is the $j$-dimensional affine subspace which contains the face $F_{j}(r \leqslant j \leqslant$ $d-1$ ), and let $\varepsilon_{j}$ be the \pm 1 coefficients associated to the sequence (2.3) with respect to $\Psi$. Let $\Psi^{\prime}$ be the $r$-flag obtained from $\Psi$ by translating all the affine subspaces in (2.5), as well as the positive and negative half-spaces, by the vector $\lambda$ (remark that it may happen that $\Psi^{\prime}$ coincides with $\Psi$ ), and let $\varepsilon_{j}^{\prime}$ be the \pm 1 coefficients associated to the sequence (2.4) with respect to $\Psi^{\prime}$. Then we have

$$
\varepsilon_{j}^{\prime}=\varepsilon_{j} \quad(r \leqslant j<d-1) \quad \text { and } \quad \varepsilon_{d-1}^{\prime}=-\varepsilon_{d-1},
$$

where the last equality is due to the fact that the facet $F_{d-1}^{\prime}$ can be obtained from $F_{d-1}$ by reflection through the center of symmetry of $A$. This implies that

$$
\varepsilon_{r} \varepsilon_{r+1} \cdots \varepsilon_{d-1} \operatorname{Vol}_{r}\left(F_{r}\right)+\varepsilon_{r}^{\prime} \varepsilon_{r+1}^{\prime} \cdots \varepsilon_{d-1}^{\prime} \operatorname{Vol}_{r}\left(F_{r}^{\prime}\right)=0 .
$$

We conclude that for any given $r$-flag $\Phi(0 \leqslant r \leqslant d-1)$, the collection of all the $r$-dimensional faces of $A$ which contribute to the sum (1.5) can be partitioned into pairs, such that the joint contribution of two $r$-dimensional faces in the same pair is equal to zero. Hence the entire sum in (1.5) must be zero, and so we obtain that $H_{\Phi}(A, L)=0$ as we had to show.

Remark 2.6. It can be seen from the proof above that the assertion in Corollary 2.5 remains true if we relax the assumption that the all the vertices of $A$ lie in $L$, and instead only require that each translation vector mapping a facet $F$ onto the parallel facet $F^{\prime}$ obtained by reflecting $F$ through the center of symmetry of $A$, belongs to $L$.

\section{Preliminaries}

The rest of the paper is devoted to the proofs of our results. The present section contains some preliminary material that will be used in the proofs. 
3.1. Notation. If $A$ is a subset of $\mathbb{R}^{d}$ and $\lambda$ is a vector in $\mathbb{R}^{d}$, then we denote by $A+\lambda$ the translation of $A$ by the vector $\lambda$. If $A, B$ are two subsets of $\mathbb{R}^{d}$, then $A+B$ and $A-B$ denote their set of sums and set of differences respectively.

We use $\langle\cdot, \cdot\rangle$ to denote the standard scalar product in $\mathbb{R}^{d}$.

By a (full-rank) lattice $L$ in $\mathbb{R}^{d}$ we refer to a discrete subgroup generated by $d$ linearly independent vectors. We use $\operatorname{det}(L)$ to denote the volume of some (or equivalently, of any) fundamental parallelepiped of $L$. The dual lattice $L^{*}$ is the set of all vectors $\lambda^{*} \in \mathbb{R}^{d}$ such that $\left\langle\lambda, \lambda^{*}\right\rangle \in \mathbb{Z}, \lambda \in L$.

For each $\xi \in \mathbb{R}^{d}$ we use the notation $e_{\xi}(x):=e^{2 \pi i\langle\xi, x\rangle}, x \in \mathbb{R}^{d}$.

The Fourier transform of a function $f \in L^{1}\left(\mathbb{R}^{d}\right)$ is normalized to be

$$
\widehat{f}(\xi)=\int_{\mathbb{R}^{d}} f(x) \overline{e_{\xi}(x)} d x,
$$

and similarly, the Fourier transform of a finite, complex measure $\mu$ on $\mathbb{R}^{d}$ is

$$
\widehat{\mu}(\xi)=\int_{\mathbb{R}^{d}} \overline{e_{\xi}(x)} d \mu(x) .
$$

3.2. The polytope group. By a polytope in $\mathbb{R}^{d}$ we mean a set which can be represented as the union of a finite number of simplices with disjoint interiors, where a simplex is the convex hull of $d+1$ points which do not all lie in some hyperplane.

It will be convenient to generalize the notion of a polytope by considering elements of the polytope group $\mathscr{P}^{d}$. We define the polytope group $\mathscr{P}^{d}$ to be the abelian group generated by the elements $[A]$ where $A$ goes through all polytopes in $\mathbb{R}^{d}$, subject to the relations $[A]+[B]=[A \cup B]$ whenever $A$ and $B$ are two polytopes with disjoint interiors. Any element $P$ of the polytope group $\mathscr{P}^{d}$ can be represented as a finite sum

$$
P=\sum_{j} m_{j}\left[A_{j}\right]
$$

where $m_{j}$ are distinct nonzero integers, and $A_{j}$ are polytopes with pairwise disjoint interiors. This representation is unique up to the order of the terms in the sum.

We think of the set of polytopes in $\mathbb{R}^{d}$ as a subset of the polytope group $\mathscr{P}^{d}$, by identifying a polytope $A$ with the element $[A]$ of $\mathscr{P}^{d}$.

A function $\varphi$, defined on the set of all polytopes in $\mathbb{R}^{d}$, is said to be additive if we have $\varphi(A \cup B)=\varphi(A)+\varphi(B)$ whenever $A$ and $B$ are two polytopes with disjoint interiors. Any such a function $\varphi$ can be extended in a unique way to a function (also denoted by $\varphi)$ on the polytope group $\mathscr{P}^{d}$, satisfying $\varphi(P)=\varphi\left(P^{\prime}\right)+\varphi\left(P^{\prime \prime}\right)$ whenever $P=P^{\prime}+P^{\prime \prime}$. The extension is given by $\varphi(P)=\sum m_{j} \varphi\left(A_{j}\right)$ for an element $P$ of the form (3.1).

For example, the $d$-dimensional volume is an additive function on the set of polytopes in $\mathbb{R}^{d}$. Hence we can extend the notion of the volume by additivity to all the elements of the polytope group $\mathscr{P}^{d}$. We will use $\operatorname{vol}(P)$ to denote the volume of an element $P \in \mathscr{P}^{d}$. Notice that $\operatorname{vol}(P)$ is a real number which may be positive, negative or zero.

We can also define, in a similar way, the indicator function $\chi_{P}$ of an element $P$ of the polytope group $\mathscr{P}^{d}$. Indeed, the mapping which takes a polytope $A$ to its indicator function $\chi_{A}$ is an additive mapping from the set of polytopes into $L^{1}\left(\mathbb{R}^{d}\right)$. Hence this mapping extends by additivity to the polytope group $\mathscr{P}^{d}$, and thus for each $P \in \mathscr{P}^{d}$ we define its indicator function $\chi_{P}$ as an element of the space $L^{1}\left(\mathbb{R}^{d}\right)$. 
For any element $P$ of the polytope group $\mathscr{P}^{d}$ we have $\int \chi_{P}(x) d x=\operatorname{vol}(P)$. Indeed, this equality is obviously true if $P$ is a polytope, and by additivity it therefore holds for all the elements of $\mathscr{P}^{d}$.

3.3. Tiling. We say that an element $P$ of the polytope group $\mathscr{P}^{d}$ tiles at level $k$ by translations with respect to a lattice $L$, if we have

$$
\sum_{\lambda \in L} \chi_{P}(x-\lambda)=k \quad \text { a.e. }
$$

Since the indicator function $\chi_{P}$ is integer-valued a.e., the tiling level $k$ is necessarily an integer, which may be positive, negative or zero.

Proposition 3.1. Let $P$ be an element of the polytope group $\mathscr{P}^{d}$, and let $L$ be a lattice in $\mathbb{R}^{d}$. If $P$ tiles at level $k$ by translations with respect to $L$, then $\operatorname{vol}(P)=k \operatorname{det}(L)$.

Proof. Let $D$ be a fundamental parallelepiped of $L$. By (3.2) we have

$$
\int_{D} \sum_{\lambda \in L} \chi_{P}(x-\lambda) d x=k \operatorname{vol}(D) \text {. }
$$

On the other hand, the left hand side of (3.3) is equal to

$$
\sum_{\lambda \in L} \int_{D} \chi_{P}(x-\lambda) d x=\sum_{\lambda \in L} \int_{D-\lambda} \chi_{P}(x) d x=\int_{\mathbb{R}^{d}} \chi_{P}(x) d x
$$

where the last equality holds since $D$ tiles (at level 1 ) by translations with respect to $L$. Since $\operatorname{vol}(D)=\operatorname{det}(L)$ and $\int \chi_{P}(x) d x=\operatorname{vol}(P)$, this proves the claim.

Proposition 3.2. Let $P$ be an element of the polytope group $\mathscr{P}^{d}$, and $L$ be a lattice in $\mathbb{R}^{d}$. Then $P$ tiles at some level $k$ by translations with respect to $L$, if and only if the function $\widehat{\chi}_{P}$ (the Fourier transform of $\chi_{P}$ ) vanishes on $L^{*} \backslash\{0\}$.

Proof. By applying a linear transformation we may suppose that $L=\mathbb{Z}^{d}$. Let

$$
f(x):=\sum_{m \in \mathbb{Z}^{d}} \chi_{P}(x-m)
$$

then $f$ is a $\mathbb{Z}^{d}$-periodic function whose Fourier series is given by

$$
\sum_{m \in \mathbb{Z}^{d}} \widehat{\chi}_{P}(m) e^{2 \pi i\langle m, x\rangle}
$$

(this is one of the variants of Poisson's summation formula, see e.g. SW71, Chapter VII, Theorem 2.4]). Hence $f$ coincides a.e. with a constant function, if and only if $\widehat{\chi}_{P}(m)=0$ for every $m \in \mathbb{Z}^{d} \backslash\{0\}$. This yields the claim.

3.4. Equidecomposability. Let $A$ and $B$ be two polytopes in $\mathbb{R}^{d}$, and let $L$ be a lattice in $\mathbb{R}^{d}$. We say that $A$ and $B$ are equidecomposable with respect to translations by vectors from $L$, if there exist finite decompositions of $A$ and $B$ of the form

$$
A=\bigcup_{j=1}^{N} A_{j}, \quad B=\bigcup_{j=1}^{N} B_{j}
$$

where $A_{1}, \ldots, A_{N}$ are polytopes with pairwise disjoint interiors, $B_{1}, \ldots, B_{N}$ are also polytopes with pairwise disjoint interiors, and $B_{j}$ can be obtained from $A_{j}$ by translation along some vector $\lambda_{j}$ belonging to $L$. 
The following proposition connects the two notions of equidecomposability and tiling by lattice translations.

Proposition 3.3. Let $A$ and $B$ be two polytopes in $\mathbb{R}^{d}$, and $L$ be a lattice in $\mathbb{R}^{d}$. Then the following two conditions are equivalent:

(i) $A$ and $B$ are equidecomposable with respect to translations by vectors from $L$;

(ii) The element $[A]-[B]$ of the polytope group $\mathscr{P}^{d}$ tiles at level zero by translations with respect to $L$.

Proof. We start by showing that (i) implies (ii). The condition (i) means that we have decompositions $[A]=\left[A_{1}\right]+\cdots+\left[A_{n}\right]$ and $[B]=\left[B_{1}\right]+\cdots+\left[B_{n}\right]$, where $A_{j}, B_{j}$ are polytopes such that $B_{j}$ is obtained from $A_{j}$ by translation along some vector $\lambda_{j} \in L$. Hence, for each $j$, the element $\left[A_{j}\right]-\left[B_{j}\right]$ is easily seen to tile at level zero by translations with respect to $L$. Since $[A]-[B]=\sum_{j=1}^{n}\left(\left[A_{j}\right]-\left[B_{j}\right]\right)$, condition (ii) follows.

Next, we prove that (ii) implies (i). The proof is done by induction on the number of elements of the set

$$
S(A, B):=\{\lambda \in L: \operatorname{vol}(A \cap(B-\lambda))>0\} .
$$

If the set $S(A, B)$ is empty, then it follows from (ii) that both $A$ and $B$ must be empty sets, so in this case the condition (i) holds trivially. Otherwise, the set $S(A, B)$ is nonempty, hence we may choose some vector $\lambda \in S(A, B)$. Then the two sets

$$
A^{\prime}:=A \cap(B-\lambda), \quad B^{\prime}:=(A+\lambda) \cap B
$$

are polytopes satisfying $B^{\prime}=A^{\prime}+\lambda$. Since $A^{\prime} \subset A$ we may decompose $A$ as the union of $A^{\prime}$ and another (possibly empty) polytope $A^{\prime \prime}$, where $A^{\prime}, A^{\prime \prime}$ have disjoint interiors. Similarly, $B$ is the union of $B^{\prime}$ and some polytope $B^{\prime \prime}$ (which, again, may be empty) such that the interiors of $B^{\prime}$ and $B^{\prime \prime}$ are disjoint. It follows from (ii) that the element $\left[A^{\prime \prime}\right]-\left[B^{\prime \prime}\right]$ of the polytope group $\mathscr{P}^{d}$ tiles at level zero by translations with respect to $L$. Moreover, the set $S\left(A^{\prime \prime}, B^{\prime \prime}\right)$ is a subset of $S(A, B) \backslash\{\lambda\}$, and therefore it has less elements than $S(A, B)$. So the inductive hypothesis implies that $A^{\prime \prime}$ and $B^{\prime \prime}$ are equidecomposable using translations by vectors from $L$. Hence the same is true also for $A$ and $B$, and thus (i) is established.

As a consequence of Proposition 3.3 we obtain Proposition 1.3 stated above:

Proof of Proposition 1.3. Let $A$ and $B$ be two polytopes in $\mathbb{R}^{d}$, let $L$ be a lattice in $\mathbb{R}^{d}$, and assume that $B$ tiles at some level $k$ by translations with respect to $L$. Then the translates of $A$ along $L$ is also a $k$-tiling, if and only if the element $[A]-[B]$ of the polytope group $\mathscr{P}^{d}$ tiles at level zero by translations along $L$. By Proposition 3.3 , the latter condition is equivalent to $A$ and $B$ being equidecomposable with respect to translations by vectors from $L$.

We also use Proposition 3.3 to establish the following claim:

Proposition 3.4. Let $P$ be an element of the polytope group $\mathscr{P}^{d}$, and let $L$ be a lattice in $\mathbb{R}^{d}$. Then $P$ tiles at level zero by translations with respect to $L$, if and only if $P$ can be represented as a finite sum $P=\sum\left(\left[A_{j}\right]-\left[A_{j}^{\prime}\right]\right)$ where $A_{j}, A_{j}^{\prime}$ are polytopes such that $A_{j}^{\prime}$ is obtained from $A_{j}$ by translation along a vector $\lambda_{j} \in L$. 
Proof. The "if" part of the claim is obvious, so we will just prove the "only if" part. Let $\mathscr{C}^{d}(L)$ denote the subgroup of the polytope group $\mathscr{P}^{d}$ generated by all the elements of the form $[A]-\left[A^{\prime}\right]$, such that $A, A^{\prime}$ are polytopes and $A^{\prime}$ is obtained from $A$ by translation along a vector from $L$. It is enough to show that if $P$ tiles at level zero by translations with respect to $L$, then $P$ belongs to the subgroup $\mathscr{C}^{d}(L)$.

The polytope group $\mathscr{P}^{d}$ is generated by the elements $[A]$, where $A$ goes through all the polytopes. We can therefore represent $P$ in the form

$$
P=\left[A_{1}\right]+\cdots+\left[A_{n}\right]-\left[B_{1}\right]-\cdots-\left[B_{m}\right],
$$

where $A_{j}, B_{k}$ are polytopes. For each $j$ we let $A_{j}^{\prime}$ be a polytope obtained from $A_{j}$ by translation along some vector in $L$, where the translation vectors are chosen such that the polytopes $A_{1}^{\prime}, \ldots, A_{n}^{\prime}$ have pairwise disjoint interiors. Hence $\left[A_{1}^{\prime}\right]+\cdots+\left[A_{n}^{\prime}\right]=\left[A^{\prime}\right]$ for a certain polytope $A^{\prime}$. In the same way, for each $k$ we let $B_{k}^{\prime}$ be a polytope obtained from $B_{k}$ by translation along some vector in $L$, such that $\left[B_{1}^{\prime}\right]+\cdots+\left[B_{m}^{\prime}\right]=\left[B^{\prime}\right]$ for some polytope $B^{\prime}$. Let $P^{\prime}:=\left[A^{\prime}\right]-\left[B^{\prime}\right]$, then

$$
P-P^{\prime}=\sum_{j=1}^{n}\left(\left[A_{j}\right]-\left[A_{j}^{\prime}\right]\right)-\sum_{k=1}^{m}\left(\left[B_{k}\right]-\left[B_{k}^{\prime}\right]\right),
$$

which shows that $P-P^{\prime}$ belongs to the subgroup $\mathscr{C}^{d}(L)$. In particular, this implies that $P-P^{\prime}$ tiles at level zero by translations with respect to $L$. Hence also $P^{\prime}=P-\left(P-P^{\prime}\right)$ tiles at level zero by translations with respect to $L$. By Proposition 3.3, the polytopes $A^{\prime}$ and $B^{\prime}$ are therefore equidecomposable with respect to translations by vectors from $L$. This implies that $P^{\prime}$ belongs to the subgroup $\mathscr{C}^{d}(L)$. We obtain that both $P^{\prime}$ and $P-P^{\prime}$ are in $\mathscr{C}^{d}(L)$, and therefore $P$ is in $\mathscr{C}^{d}(L)$ as well, as we had to show.

3.5. Flags. If $r$ is an integer, $0 \leqslant r \leqslant d-1$, then an $r$-flag $\Phi$ in $\mathbb{R}^{d}$ is defined to be a sequence of affine subspaces

$$
V_{r} \subset V_{r+1} \subset \cdots \subset V_{d-1} \subset V_{d}=\mathbb{R}^{d}
$$

such that $V_{j}$ has dimension $j$. Each subspace $V_{j}(r \leqslant j \leqslant d-1)$ in the sequence divides the next one $V_{j+1}$ into two half-spaces; we assume that $\Phi$ is endowed with a choice of one of these half-spaces being called positive, and the other being called negative.

It will be convenient to define also a $d$-flag in $\mathbb{R}^{d}$ to be the sequence which consists of just one subspace $V_{d}=\mathbb{R}^{d}$.

Let $A$ be a polytope in $\mathbb{R}^{d}$, and suppose that we have a sequence

$$
F_{r} \subset F_{r+1} \subset \cdots \subset F_{d-1} \subset F_{d}=A,
$$

where $F_{j}$ is a $j$-dimensional face of $A(r \leqslant j \leqslant d-1)$. Such a sequence will be called an $r$-sequence of faces of the polytope $A$, and will be denoted by $\mathscr{F}_{r}$.

We will say that the $r$-sequence $\mathscr{F}_{r}$ is contained in the $r$-flag $\Phi$ if the face $F_{j}$ is contained in the subspace $V_{j}$ for each $r \leqslant j \leqslant d-1$.

Each $r$-flag $\Phi(0 \leqslant r \leqslant d)$ determines an additive "weight" function $\omega_{\Phi}$, which is defined by (1.4) on the set of polytopes, and is extended by additivity to the polytope group $\mathscr{P}^{d}$. Notice that if $\Phi$ is a $d$-flag, then $\omega_{\Phi}(P)=\operatorname{vol}(P)$ for every $P \in \mathscr{P}^{d}$.

The Hadwiger functional $H_{\Phi}(\cdot, L)$ associated to an $r$-flag $\Phi(0 \leqslant r \leqslant d)$ and to a lattice $L$ in $\mathbb{R}^{d}$, is the additive function on the polytope group $\mathscr{P}^{d}$ which is given by (1.5) on the set of polytopes. The Hadwiger functional is invariant with respect to 
translations by vectors from $L$, that is, $H_{\Phi}(P, L)=H_{\Phi}(Q, L)$ if $Q$ is obtained from $P$ by translation along a vector $\lambda \in L$.

3.6. Flag measures. Let $\Phi$ be an $r$-flag in $\mathbb{R}^{d}(0 \leqslant r \leqslant d)$, determined by a sequence of affine subspaces (3.4). For each polytope $A$ we define a signed measure $\nu_{A, \Phi}$ on $\mathbb{R}^{d}$ given by

$$
\nu_{A, \Phi}=\left.\sum_{\mathscr{F}_{r}} \varepsilon_{r} \varepsilon_{r+1} \cdots \varepsilon_{d-1} \operatorname{Vol}_{r}\right|_{F_{r}}
$$

where $\mathscr{F}_{r}$ goes through all $r$-sequences of faces $F_{r} \subset F_{r+1} \subset \cdots \subset F_{d}$ of the polytope $A$ that are contained in $\Phi$, the $\varepsilon_{j}$ are the \pm 1 coefficients associated to the $r$-sequence $\mathscr{F}_{r}$ with respect to $\Phi$ in the same way as in (1.4), and where $\left.\operatorname{Vol}_{r}\right|_{F_{r}}$ denotes the $r$ dimensional volume measure restricted to the face $F_{r}$.

In the case when $r=0$, by a 0 -dimensional face of $A$ we mean a vertex of $A$, and the measure $\left.\operatorname{Vol}_{r}\right|_{F_{r}}$ is understood to be the Dirac measure at the vertex $F_{r}$.

The mapping which takes a polytope $A$ to the measure $\nu_{A, \Phi}$ can be seen to be an additive mapping, that is, $\nu_{A \cup B, \Phi}=\nu_{A, \Phi}+\nu_{B, \Phi}$ whenever $A$ and $B$ are two polytopes with disjoint interiors. Hence we can extend this mapping by additivity, and define the signed measure $\nu_{P, \Phi}$ for any element $P$ of the polytope group $\mathscr{P}^{d}$.

Remark that if $\Phi$ is a $d$-flag, then $\nu_{P, \Phi}$ is the measure $\chi_{P}(x) d x$, that is, an absolutely continuous measure with density $\chi_{P}$ with respect to the Lebesgue measure $d x$.

It follows from (1.4) and (3.5) that the measure $\nu_{P, \Phi}$ satisfies $\int d \nu_{P, \Phi}=\omega_{\Phi}(P)$, for any $r$-flag $\Phi(0 \leqslant r \leqslant d)$ and any element $P$ of the polytope group $\mathscr{P}^{d}$.

If $\Phi$ is an $r$-flag in $\mathbb{R}^{d}(0 \leqslant r \leqslant d), L$ is a lattice in $\mathbb{R}^{d}$, and $P$ is an element of the polytope group $\mathscr{P}^{d}$, then we define the signed measure

$$
\mu_{P, \Phi, L}=\sum_{\Psi} \nu_{P, \Psi}
$$

where $\Psi$ runs through all $r$-flags for which there exists $\lambda \in L$ such that $\Psi$ can be obtained from $\Phi$ by translating all the affine subspaces in (3.4), as well as the positive and negative half-spaces, by the vector $\lambda$. As a function of $P$, the measure $\mu_{P, \Phi, L}$ is additive and invariant under translations by vectors from $L$. It satisfies

$$
\int d \mu_{P, \Phi, L}=H_{\Phi}(P, L)
$$

\section{Proof of main Result}

4.1. In this section we prove the following theorem:

Theorem 4.1. Let $P$ be an element of the polytope group $\mathscr{P}^{d}$, and let $L$ be a lattice in $\mathbb{R}^{d}$. Then $P$ tiles at some level $k$ by translations with respect to $L$, if and only if $H_{\Phi}(P, L)=0$ for every $r$-flag $\Phi(0 \leqslant r \leqslant d-1)$.

This is, in fact, the main result of this paper. It generalizes the statement of Theorem 1.1. from the set of polytopes to all the elements of the polytope group $\mathscr{P}^{d}$. In particular, Theorem 1.1 follows from Theorem 4.1 in the special case when $P$ is a polytope.

We also deduce Theorem 1.2 from Theorem 4.1. This is done as follows: 
Proof of Theorem 1.2. Let $A$ and $B$ be two polytopes in $\mathbb{R}^{d}$, and let $L$ be a lattice in $\mathbb{R}^{d}$. If $A$ and $B$ are equidecomposable with respect to translations by vectors from $L$, then it is obvious that $A$ and $B$ have the same volume, and

$$
H_{\Phi}(A, L)=H_{\Phi}(B, L)
$$

for every $r$-flag $\Phi(0 \leqslant r \leqslant d-1)$. This is so because the volume function, as well as all the Hadwiger functionals $H_{\Phi}(\cdot, L)$, are additive functions on the set of polytopes that are invariant with respect to translations by vectors from $L$.

Conversely, suppose that $A$ and $B$ have the same volume, and that (4.1) holds for every $r$-flag $\Phi(0 \leqslant r \leqslant d-1)$. Then by additivity, the element $P:=[A]-[B]$ of the polytope group $\mathscr{P}^{d}$ has volume zero, and it satisfies $H_{\Phi}(P, L)=0$ for every $r$-flag $\Phi$ $(0 \leqslant r \leqslant d-1)$. Theorem 4.1 therefore yields that $P$ tiles at some level $k$ by translations with respect to $L$. By Proposition 3.1 we have $\operatorname{vol}(P)=k \operatorname{det}(L)$, hence the tiling level $k$ must be zero. We can therefore invoke Proposition 3.3, which yields that $A$ and $B$ are equidecomposable with respect to translations by vectors from $L$.

4.2. The remainder of this section is devoted to the proof of Theorem 4.1. The next lemma is the key one in the proof:

Lemma 4.2. Let $P$ be an element of the polytope group $\mathscr{P}$, let $L$ be a lattice in $\mathbb{R}^{d}$, and let $r$ be an integer, $0 \leqslant r \leqslant d-1$. Assume that $H_{\Psi}(P, L)=0$ for every $s$-flag $\Psi$ $(0 \leqslant s \leqslant r)$. Then for every $r$-flag $\Phi$ we have

$$
\widehat{\mu}_{P, \Phi, L}(\xi)=0
$$

for all $\xi \in L^{*}$.

Proof. Let $\Phi$ be an $r$-flag determined by a sequence of affine subspaces

$$
V_{r} \subset V_{r+1} \subset \cdots \subset V_{d-1} \subset V_{d}=\mathbb{R}^{d},
$$

such that $V_{j}$ has dimension $j$. We must show that (4.2) holds for all $\xi \in L^{*}$. We prove this by induction on $r$.

By applying a translation we may assume that all the affine subspaces in (4.3) are in fact linear subspaces. We will first show that (4.2) is true for every $\xi \in L^{*} \cap V_{r}^{\perp}$, where $V_{r}^{\perp}$ denotes the orthogonal complement of the linear subspace $V_{r}$. Indeed, for such $\xi$ we have $e_{\xi}(x)=1$ for all $x$ belonging to the support of the measure $\mu_{P, \Phi, L}$, since the support of $\mu_{P, \Phi, L}$ is contained in the set $V_{r}+L$ and we have $\langle x, \xi\rangle \in \mathbb{Z}$ whenever $x$ is a point in $V_{r}+L$. This implies that

$$
\widehat{\mu}_{P, \Phi, L}(\xi)=\int \overline{e_{\xi}} d \mu_{P, \Phi, L}=\int d \mu_{P, \Phi, L}=H_{\Phi}(P, L) .
$$

Hence (4.2) follows using the assumption that $H_{\Phi}(P, L)=0$.

Notice that if $r=0$, then the subspace $V_{r}^{\perp}$ coincides with the whole space $\mathbb{R}^{d}$. So in this case, the full assertion of Lemma 4.2 is already established. Hence, in what follows we will assume that $r \geqslant 1$. In this case, it still remains for us to show that (4.2) is true also for every $\xi \in L^{*} \backslash V_{r}^{\perp}$.

The polytope group $\mathscr{P}^{d}$ is generated by the elements $[A]$, where $A$ goes through all the polytopes in $\mathbb{R}^{d}$. We can therefore represent $P$ as a finite sum $P=\sum m_{k}\left[A_{k}\right]$, where $m_{k}$ are integers and $A_{k}$ are polytopes. By additivity we have

$$
\mu_{P, \Phi, L}=\sum_{k} m_{k} \mu_{A_{k}, \Phi, L}
$$


The Fourier transform of the measure $\mu_{A_{k}, \Phi, L}$ at the point $\xi$ is given by

$$
\widehat{\mu}_{A_{k}, \Phi, L}(\xi)=\int \overline{e_{\xi}} d \mu_{A_{k}, \Phi, L}=\sum_{\Psi} \sum_{\mathscr{F}_{r}} \varepsilon_{r} \varepsilon_{r+1} \cdots \varepsilon_{d-1} \int_{F_{r}} \overline{e_{\xi}},
$$

where $\Psi$ runs through all $r$-flags that can be obtained from $\Phi$ by translation along a vector from $L, \mathscr{F}_{r}$ goes through all $r$-sequences of faces $F_{r} \subset F_{r+1} \subset \cdots \subset F_{d}$ of the polytope $A_{k}$ that are contained in $\Psi$, the $\varepsilon_{j}$ 's are the \pm 1 coefficients associated to the $r$-sequence $\mathscr{F}_{r}$ with respect to $\Psi$, and the integral on the right hand side is taken with respect to the $r$-dimensional volume measure on the face $F_{r}$.

Let $\partial F_{r}$ denote the relative boundary of the face $F_{r}$, and for each $x \in \partial F_{r}$ let $n(x)$ be a vector in the linear subspace $V_{r}$ which is outward unit normal to $F_{r}$ at the point $x$. Then for every $v \in V_{r}$ we have

$$
-2 \pi i\langle\xi, v\rangle \int_{F_{r}} \overline{e_{\xi}}=\int_{\partial F_{r}}\langle n, v\rangle \overline{e_{\xi}},
$$

which follows by applying the divergence theorem to the function $f(x)=v \overline{e_{\xi}(x)}$ over the face $F_{r}$. The relative boundary $\partial F_{r}$ consists of a finite number of $(r-1)$-dimensional faces $F_{r-1}$ of $F_{r}$. Hence, using (4.6) and (4.7), we get

$$
\begin{gathered}
-2 \pi i\langle\xi, v\rangle \widehat{\mu}_{A_{k}, \Phi, L}(\xi)=\sum_{\Psi} \sum_{\mathscr{F}_{r}} \varepsilon_{r} \varepsilon_{r+1} \cdots \varepsilon_{d-1} \int_{\partial F_{r}}\langle n, v\rangle \overline{e_{\xi}} \\
=\sum_{\Psi} \sum_{\mathscr{F}_{r}} \varepsilon_{r} \varepsilon_{r+1} \cdots \varepsilon_{d-1} \sum_{F_{r-1}}\langle n, v\rangle \int_{F_{r-1}} \overline{e_{\xi}},
\end{gathered}
$$

where $F_{r-1}$ goes through the $(r-1)$-dimensional faces of the $r$-dimensional face $F_{r}$ from the sequence $\mathscr{F}_{r}$, and $n$ is the outward unit normal to $F_{r}$ on $F_{r-1}$.

Let $\mathscr{E}$ be the collection of all the $(r-1)$-sequences of faces $F_{r-1} \subset F_{r} \subset \cdots \subset F_{d}$ that belong to one of the polytopes $A_{k}$ (where $k$ may be different from one sequence to another, so that $k$ is not fixed), and such that the $r$-subsequence $F_{r} \subset F_{r+1} \subset \cdots \subset F_{d}$ is contained in some $r$-flag $\Psi$ that can be obtained from $\Phi$ by translation along a vector from $L$. We define an equivalence relation on $\mathscr{E}$ by saying that two elements $\mathscr{F}_{r-1}$ and $\mathscr{F}_{r-1}^{\prime}$ from $\mathscr{E}$ are equivalent if the affine hull of the $(r-1)$-dimensional face $F_{r-1}$ from the sequence $\mathscr{F}_{r-1}$ can be translated by a vector in $L$ onto the affine hull of the $(r-1)$ dimensional face $F_{r-1}^{\prime}$ from $\mathscr{F}_{r-1}^{\prime}$. Then $\mathscr{E}$ can be partitioned into a finite number of equivalence classes $\mathscr{E}^{1}, \mathscr{E}^{2}, \ldots, \mathscr{E}^{N}$ induced by this equivalence relation.

To each equivalence class $\mathscr{E}^{l}(1 \leqslant l \leqslant N)$ we associate an $(r-1)$-flag $\Phi^{l}$, defined in the following way. The flag $\Phi^{l}$ is determined by a sequence of affine subspaces

$$
V_{r-1}^{l} \subset V_{r} \subset V_{r+1} \subset \cdots \subset V_{d}=\mathbb{R}^{d},
$$

where $V_{r}, V_{r+1}, \ldots, V_{d}$ are the same linear subspaces from (4.3) that determine the $r$ flag $\Phi$, while $V_{r-1}^{l}$ is a new affine subspace of dimension $r-1$. The subspace $V_{r-1}^{l}$ is chosen in such a way that the set $V_{r-1}^{l}+L$ contains all the $(r-1)$-dimensional faces $F_{r-1}$ belonging to sequences $\mathscr{F}_{r-1}$ from the equivalence class $\mathscr{E} l$. It is straightforward to verify that such a choice of $V_{r-1}^{l}$ is indeed possible, thanks to the definition of the collection $\mathscr{E}$ and the way in which the equivalence relation on $\mathscr{E}$ was defined. We endow the $(r-1)$-flag $\Phi^{l}$ with a choice of positive and negative half-spaces, by saying that the positive and negative half-spaces of $V_{j+1}$ determined by the subspace $V_{j}$ coincide with 
those from the $r$-flag $\Phi$ for all $r \leqslant j \leqslant d-1$; while the positive and negative half-spaces of $V_{r}$ that are determined by the new subspace $V_{r-1}^{l}$ are selected in an arbitrary way.

For each $1 \leqslant l \leqslant N$, let $\sigma^{l}$ denote the (unique) unit vector in the linear subspace $V_{r}$ which is normal to $V_{r-1}^{l}$ and is pointing towards the negative half-space of $V_{r}$ determined by $V_{r-1}^{l}$. We then observe that for any given $k$, the sum in (4.9) is equal to

$$
\sum_{l=1}^{N}\left\langle\sigma^{l}, v\right\rangle \sum_{\Theta} \sum_{\mathscr{F}_{r-1}} \varepsilon_{r-1} \varepsilon_{r} \varepsilon_{r+1} \cdots \varepsilon_{d-1} \int_{F_{r-1}} \overline{e_{\xi}},
$$

where $\Theta$ runs through all $(r-1)$-flags that can be obtained from $\Phi^{l}$ by translation along a vector from $L, \mathscr{F}_{r-1}$ goes through all $(r-1)$-sequences of faces $F_{r-1} \subset F_{r} \subset \cdots \subset F_{d}$ of the polytope $A_{k}$ that are contained in $\Theta$, and the $\varepsilon_{j}$ 's are the \pm 1 coefficients associated to the $(r-1)$-sequence $\mathscr{F}_{r-1}$ with respect to $\Theta$. Hence (4.8), (4.9) and (4.10) give that

$$
-2 \pi i\langle\xi, v\rangle \widehat{\mu}_{A_{k}, \Phi, L}(\xi)=\sum_{l=1}^{N}\left\langle\sigma^{l}, v\right\rangle \widehat{\mu}_{A_{k}, \Phi^{l}, L}(\xi),
$$

for each $k$. Finally, by multiplying both sides of (4.11) by $m_{k}$ and then taking the sum with respect to $k$, we conclude that

$$
-2 \pi i\langle\xi, v\rangle \widehat{\mu}_{P, \Phi, L}(\xi)=\sum_{l=1}^{N}\left\langle\sigma^{l}, v\right\rangle \widehat{\mu}_{P, \Phi^{l}, L}(\xi)
$$

for every $\xi \in \mathbb{R}^{d}$ and every $v \in V_{r}$.

Now recall that it remained to show that (4.2) is true for every $\xi \in L^{*} \backslash V_{r}^{\perp}$. Since $\xi \in L^{*}$, it follows from the inductive hypothesis that $\widehat{\mu}_{P, \Phi^{l}, L}(\xi)=0$ for each $1 \leqslant l \leqslant N$. On the other hand, since $\xi \notin V_{r}^{\perp}$ there exists a vector $v \in V_{r}$ such that $\langle\xi, v\rangle \neq 0$. Hence (4.2) follows from the equality (4.12), and so the proof is complete.

4.3. Based on Lemma 4.2 we next establish the following result:

Lemma 4.3. Let $P$ be an element of the polytope group $\mathscr{P}^{d}$, and let $L$ be a lattice in $\mathbb{R}^{d}$. Assume that $H_{\Psi}(P, L)=0$ for every $s$-flag $\Psi(0 \leqslant s \leqslant d-1)$. Then $\widehat{\chi}_{P}(\xi)=0$ for all $\xi \in L^{*} \backslash\{0\}$.

Proof. Recall that by a $d$-flag we mean the sequence which consists of just one subspace $V_{d}=\mathbb{R}^{d}$. If $\Phi$ is a $d$-flag, then the measure $\mu_{P, \Phi, L}$ is equal to $\chi_{P}(x) d x$ (that is, an absolutely continuous measure with density $\left.\chi_{P}\right)$. Hence, the assertion of Lemma 4.3 can be equivalently stated by saying that (4.2) holds for all $\xi \in L^{*} \backslash V_{d}^{\perp}$.

The arguments in the proof of Lemma 4.2 are valid also in the case when $r=d$. In particular this is true for the second part of the proof, where it is shown (based on the inductive hypothesis) that (4.2) holds for every $\xi \in L^{*} \backslash V_{r}^{\perp}$. Notice that the assumption that $H_{\Psi}(P, L)=0$ for every $s$-flag $\Psi$ was used in that part of the proof only for $0 \leqslant s \leqslant r-1$ (and not for $s=r$ ). Hence the proof applies to our present situation as well, and allows us to conclude that (4.2) is indeed true for all $\xi \in L^{*} \backslash\{0\}$.

\subsection{We can now finish the proof of Theorem 4.1:}

Proof of Theorem 4.1. Assume first that $P$ tiles at level $k$ by translations with respect to $L$. Let $D$ be a fundamental parallelepiped of $L$, then the element $P^{\prime}:=P-k[D]$ of $\mathscr{P}^{d}$ tiles at level zero by translations with respect to $L$. Hence by Proposition 3.4 we 
can represent $P^{\prime}$ as a finite sum $P^{\prime}=\sum\left(\left[A_{j}\right]-\left[A_{j}^{\prime}\right]\right)$ where $A_{j}, A_{j}^{\prime}$ are polytopes such that $A_{j}^{\prime}$ is obtained from $A_{j}$ by translation along a vector from $L$. Since the Hadwiger functionals $H_{\Phi}(\cdot, L)$ are additive and invariant with respect to translations by vectors from $L$, it follows that $H_{\Phi}\left(P^{\prime}, L\right)=0$ for every $r$-flag $\Phi(0 \leqslant r \leqslant d-1)$. It is also easy to check that $H_{\Phi}(D, L)=0$ for every such $\Phi$ (in fact, this follows from the proof of Corollary 2.5). Since $P=P^{\prime}+k[D]$, using additivity again implies that $H_{\Phi}(P, L)=0$ for every $\Phi$, which proves one part of Theorem 4.1 .

To prove the converse part, suppose that $H_{\Phi}(P, L)=0$ for every $r$-flag $\Phi(0 \leqslant r \leqslant$ $d-1)$. Then we apply Lemma 4.3 and obtain that $\widehat{\chi}_{P}(\xi)=0$ for all $\xi \in L^{*} \backslash\{0\}$. This implies, by Proposition 3.2 , that $P$ tiles at some level $k$ by translations with respect to $L$, and thus concludes the proof of Theorem 4.1 .

\section{REMARK}

Equidecomposability using translations by vectors from a lattice $L$, can easily be seen to constitute an equivalence relation on the set of all polytopes in $\mathbb{R}^{d}$. Theorem 1.2 characterizes the polytopes which lie in the same equivalence class with respect to this relation: two polytopes $A$ and $B$ are equidecomposable using translations by vectors from $L$, if and only if $H_{\Phi}(A, L)=H_{\Phi}(B, L)$ for every $r$-flag $\Phi(0 \leqslant r \leqslant d)$.

(We remind the reader that the meaning of the latter condition in the case when $r=d$ is that $A$ and $B$ have the same volume.)

The condition that $H_{\Phi}(P, L)=H_{\Phi}(Q, L)$ for every $\Phi$, in fact makes sense for any two elements $P$ and $Q$ of the polytope group $\mathscr{P}^{d}$. This extends the equivalence relation described above to all the elements of the polytope group $\mathscr{P}^{d}$. One may thus wonder whether the equivalence classes with respect to this relation admit any geometric description, in a way that generalizes the notion of equidecomposability of polytopes.

Based on Theorem 4.1, we can obtain an affirmative answer to this question. The following version of Theorem 1.2 is true:

Theorem 5.1. Let $P$ and $Q$ be two elements of the polytope group $\mathscr{P} d$, and let $L$ be a lattice in $\mathbb{R}^{d}$. Then the following two conditions are equivalent:

(i) $P$ and $Q$ can be represented in the form

$$
P=R+\left[A_{1}\right]+\cdots+\left[A_{n}\right], \quad Q=R+\left[B_{1}\right]+\cdots+\left[B_{n}\right],
$$

where $R$ is an element of the polytope group $\mathscr{P}^{d}, A_{j}, B_{j}$ are polytopes, and $B_{j}$ can be obtained from $A_{j}$ by translation along some vector $\lambda_{j} \in L$;

(ii) $H_{\Phi}(P, L)=H_{\Phi}(Q, L)$ for every $r$-flag $\Phi(0 \leqslant r \leqslant d)$.

Proof. Since the Hadwiger functionals $H_{\Phi}(\cdot, L)$ are additive and invariant with respect to translations by vectors from $L$, it is obvious that (i) implies (ii). In order to prove the converse, suppose that (ii) holds. Then by additivity we have $H_{\Phi}(P-Q, L)=0$ for every $r$-flag $\Phi(0 \leqslant r \leqslant d)$. Using Theorem 4.1 we deduce that $P-Q$ tiles at some level $k$ by translations with respect to $L$; and since $P-Q$ has volume zero, it follows from Proposition 3.1 that the tiling level $k$ must be zero. By Proposition 3.4 we can therefore represent $P-Q$ in the form $P-Q=\sum_{j=1}^{n}\left(\left[A_{j}\right]-\left[B_{j}\right]\right)$, where $A_{j}, B_{j}$ are polytopes such that $B_{j}$ is obtained from $A_{j}$ by translation along a vector from $L$. Hence (5.1) can be satisfied by taking $R=P-\sum\left[A_{j}\right]=Q-\sum\left[B_{j}\right]$, so condition (i) is established. 
Remark 5.2. Theorem 5.1 can be viewed as a version of Theorem 1.2 that applies to all the elements of the polytope group $\mathscr{P}^{d}$ (and not just to polytopes). However, notice that in the special case when $P$ and $Q$ are polytopes, Theorem 5.1 gives a weaker result than Theorem 1.2. This is because the condition (i) in Theorem 5.1 does not say that if $P$ and $Q$ are polytopes then the element $R$ in (5.1) can in fact be chosen to be zero.

\section{REFERENCES}

[Bol94] U. Bolle, On multiple tiles in $E^{2}$. Intuitive geometry (Szeged, 1991), 39-43, Colloq. Math. Soc. János Bolyai, 63, North-Holland, Amsterdam, 1994.

[Bol78] V. Boltianski, Hilbert's third problem. Wiley, 1978.

[Deh01] M. Dehn, Ueber den Rauminhalt. Math. Ann. 55 (1901), no. 3, 465-478 (German).

[GKRS13] N. Gravin, M. Kolountzakis, S. Robins, D. Shiryaev, Structure results for multiple tilings in 3D. Discrete Comput. Geom. 50 (2013), no. 4, 1033-1050.

[GRS12] N. Gravin, S. Robins, D. Shiryaev, Translational tilings by a polytope, with multiplicity. Combinatorica 32 (2012), no. 6, 629-649.

[GL15] S. Grepstad, N. Lev, Sets of bounded discrepancy for multi-dimensional irrational rotation. Geom. Funct. Anal. 25 (2015), no. 1, 87-133.

[Had52] H. Hadwiger, Translationsinvariante, additive und schwachstetige Polyederfunktionale. Arch. Math. 3 (1952), 387-394 (German).

[Had54] H. Hadwiger, Zum Problem der Zerlegungsgleichheit k-dimensionaler Polyeder. Math. Ann. 127 (1954), 170-174 (German).

[Had57] H. Hadwiger, Vorlesungen über Inhalt, Oberfläche und Isoperimetrie. Springer-Verlag, 1957 (German).

[Had68] H. Hadwiger, Translative Zerlegungsgleichheit der Polyeder des gewöhnlichen Raumes. J. Reine Angew. Math. 233 (1968), 200-212 (German).

[HG51] H. Hadwiger, P. Glur, Zerlegungsgleichheit ebener Polygone. Elem. Math. 6 (1951), 97-106 (German).

[Jes72] B. Jessen, Zur Algebra der Polytope, Nachr. Akad. Wiss. Göttingen Math.-Phys. Kl. II (1972), 47-53 (German).

[JT78] B. Jessen, A. Thorup, The algebra of polytopes in affine spaces. Math. Scand. 43 (1978), 211-240.

[Kol00] M. Kolountzakis, On the structure of multiple translational tilings by polygonal regions. Discrete Comput. Geom. 23 (2000), no. 4, 537-553.

[LM95a] J. C. Lagarias, D. Moews, Polytopes that fill $\mathbb{R}^{n}$ and scissors congruence. Discrete Comput. Geom. 13 (1995), no. 3-4, 573-583.

[LM95b] J. C. Lagarias, D. Moews, Acknowledgment of priority concerning: "Polytopes that fill $\mathbb{R}^{n}$ and scissors congruence". Discrete Comput. Geom. 14 (1995), no. 3, 359-360.

[Mür75] P. Mürner, Translative Parkettierungspolyeder und Zerlegungsgleichheit. Elem. Math. 30 (1975), 25-27 (German).

[Mür77] P. Mürner, Translative Zerlegungsgleichheit von Polytopen. Arch. Math. 29 (1977), 218-224 (German).

[Sah79] C.-H. Sah, Hilbert's third problem: scissors congruence. Research Notes in Mathematics. 33. Pitman Advanced Publishing Program, San Francisco, 1979.

[SW71] E. M. Stein, G. Weiss, Introduction to Fourier analysis on Euclidean spaces. Princeton University Press, 1971.

[Syd65] J.-P. Sydler, Conditions nécessaires et suffisantes pour l'équivalence des polyèdres de l'espace euclidien à trois dimensions. Comment. Math. Helv. 40 (1965), 43-80 (French).

Department of MATHEMATiCs, BAR-Ilan University, RAMAT-GAN 5290002, IsRAeL

E-mail address: levnir@math.biu.ac.il

Department of Mathematics, Bar-Ilan University, RAMAT-Gan 5290002, Israel

E-mail address: bochen.1iu1989@gmail.com 